\title{
Complete Remission of Generalized Myasthenia Gravis by Corticosteroid Treatment Alone without Thymectomy
}

\author{
Hiroshi Kataoka Yoshiko Furiya Satoshi Ueno \\ Departments of Neurology, Nara Medical University, Kashihara, Japan
}

\section{Key Words}

Myasthenia gravis · Steroid · Corticosteroid treatment · Thymectomy · Immunotherapy

\begin{abstract}
Many long-term, follow-up studies have shown that steroids are effective in patients with myasthenia gravis (MG) who have undergone thymectomy. However, few long-term studies have documented the response of MG to steroids alone. We describe two patients who successfully resolved the symptoms of MG without myasthenic crisis or serious side effects by steroid treatment without thymectomy or other alternative therapies for more than 19 years. Our experiences raise an open question of the role of thymectomy or steroid treatment alone, especially in MG patients without thymoma.
\end{abstract}

\section{Introduction}

Steroids have been widely used for the treatment of myasthenia gravis (MG) since the 1950s, being first introduced in 1935 [1]. Steroids are often considered as an alternative means of immunotherapy because other immunologic treatments such as plasma exchange, intravenous immunoglobulins, or immunosuppressive agents are expensive and associated with potentially serious adverse effects. Many long-term, follow-up studies have shown that steroids are effective in patients with $M G$ who have undergone thymectomy or who concurrently receive immunosuppressive agents or plasma exchange. However, few long-term studies have documented the response of MG to steroids alone [2-4]. It is therefore of great interest whether monotherapy with steroids is effective in MG. We describe two patients with generalized MG whose disease was controlled for more than 19 years by treatment with steroids alone. 


\section{Case Reports}

\section{Patient 1}

In September 2002, a 34-year-old woman with a history of MG visited our hospital. She was not receiving medication. At 6 years of age, a neurologist was consulted because of bilateral ptosis and diplopia. The results of an edrophonium test were positive. The patient was given a diagnosis of MG and administered oral prednisolone, which reduced the disease severity. Similar symptoms often developed subsequently, and the dose of prednisolone was adjusted as required. At 8 years of age, the patient presented with general weakness with respiratory dysfunction and received 3 courses of pulse therapy with intravenous methylprednisolone. At the age of 12 years, oral prednisolone was discontinued. She gave birth without symptoms of MG at the age of 33 years. In January 2004, she noticed diplopia and ptosis with general fatigue, particularity in the evening. Moderately limited ocular motility was present. There was no other neurological deficit. Grasping power decreased from $12 \mathrm{~kg}$ to 6 $\mathrm{kg}$ after she gripped the hand dynamometer 5 times. The results of a routine serum laboratory workup were normal. The anti-acetylcholine receptor-binding antibody titer was $2.3 \mathrm{nmol} / \mathrm{l}(>0.2 \mathrm{nmol} / \mathrm{l}$; normal upper limit). A computed tomographic scan of the chest showed no evidence of mediastinal thymoma. Cholinesterase inhibitor treatment was started. In September 2004, she had difficulty in swallowing saliva and holding her child. Daily oral prednisolone ( 5 to $25 \mathrm{mg} /$ day) was started in combination with a fixed dose of cholinesterase inhibitor. The severity of disease improved. Subsequently, the patient continued to receive oral prednisolone because mildly limited oculomotor motility persisted. In April 2011, she was free of the MG symptoms, and treatment with prednisolone was discontinued.

\section{Patient 2}

In July 1993, a 40-year-old woman with no clinically significant past history of disease experienced difficulty in speaking for prolonged periods. In October, she noticed nasal discharge during meals and had difficulty in swallowing or chewing food. In January 1994, she visited our hospital. At presentation, a nasal voice, bulbar paresis, and mild bilateral facial weakness were evident. The results of neurological examinations were negative. The results of a routine serum laboratory workup were normal. Cranial and cervical magnetic resonance images (MRI) were normal. The anti-acetylcholine receptor-binding antibody titer was $0.6 \mathrm{nmol} / \mathrm{l}$. The edrophonium test was positive. Repetitive stimulation studies demonstrated a decremental response at slow stimulation rate in the orbicular muscle of the mouth and the abductor digiti minimi and deltoid. Mediastinal thymoma was absent on chest MRI, and MG was diagnosed. The patient received ambenonium ( $10 \mathrm{mg} /$ day), and the disease severity decreased mildly. In June 2002, speech impairment and dysphagia developed with severe general fatigue. Oral prednisolone $(60 \mathrm{mg})$ on alternate days was started, and the bulbar symptoms and facial muscle weakness resolved. Oral prednisolone was continued with tapering, with no further disease progression. The patient was free of general fatigue. In 2008, she gave birth without the development of MG symptoms. As of April 2010, the patient continues to receive alternate-day oral prednisolone (10 mg). In April 2011, the oral prednisolone was tapered. No other symptoms of MG have developed.

\section{Discussion}

Our experience suggests that steroid treatment without thymectomy or other alternative therapies, such as plasma exchange, intravenous immunoglobulins, or immunosuppressive agents, can successfully resolve the symptoms of MG in selected patients, without myasthenic crisis or serious side effects. Many previous studies have reported the remission of $\mathrm{MG}$ in patients given long-term steroid treatment, but most patients received concurrent treatments, including thymectomy. One prospective placebo-control study of mycophenolate mofetil for 36 weeks included steroid-treated patients with generalized MG who had not undergone thymectomy within 12 months or received plasma exchange, intravenous immunoglobulins, or immunosuppressive agents within 3 months before randomization [2]. Steroid treatment was shown to be effective 
for inducing remission of disease; beneficial effects of steroids required a dose of at least $20 \mathrm{mg} /$ day [2]. Another retrospective cohort study of 60 patients with generalized MG, including 10 who had received continuous steroid treatment for more than 4 years, reported that improvement was noted in $72 \%$ of the patients given steroids [3]. However, complete withdrawal of steroids was possible in only 3 patients [3]. Kawaguchi et al. [4] found that 34 out of 67 generalized MG patients without thymectomy responded to corticosteroids in at least 12 months but 32 patients had a mild generalized form of MG. The other 2 patients with a moderate generalized form of MG were not mentioned in detail [4]. The optimal dosage and treatment schedule for steroids remains undefined [5]. Many patients and physicians avoid using high doses of steroids and are highly motivated to reduce the dose whenever possible.

We used steroid pulse therapy during childhood in patient 1 because we expected a rapid onset of response to steroids and because intravenous injection was relatively noninvasive in contrast to plasmapheresis. Steroid pulse therapy has a potential risk of temporarily worsening MG, referred to as 'steroid dip', but a study published in 2011 showed that early aggressive high-dose intravenous methylprednisolone has the advantages of early improvement with less frequent steroid-related complications as compared with high-dose oral prednisolone therapy [6].

In a recent review article, S. Sathasivam recommends: 'Steroids are useful as short-term immunosuppressants, and there is limited evidence for their efficacy in MG from small randomized trials (class II evidence)' [7]. In the recent EFNS Guidelines for treatment of autoimmune neuromuscular transmission disorders, the authors state that 'In observational studies, remission or marked improvement is seen in $70-80 \%$ of patients with MG treated with oral corticosteroids (class IV evidence), but the efficacy has not been studied in double-blind, placebo-controlled trials' [8]. Our experience can raise an open question regarding the role of thymectomy, especially in the subgroup of patients without thymoma. We suggest that medical therapy is preferable to surgery in patients with no evidence of thymoma on CT or MRI who also have a low titer of antiacetylcholine receptor-binding antibody.

\section{References}

1 Simon HE: Myasthenia gravis: effect of treatment with anterior pituitary extract. JAMA 1935;104:2065-2066.

12 Muscle Study Group: A trial of mycophenolate mofetil with prednisone as initial immunotherapy in myasthenia gravis. Neurology 2008;71:394-399.

-3 Sghirlanzoni A, Peluchetti D, Mantegazza R, Fiacchino F, Cornelio F: Myasthenia gravis: prolonged treatment with steroids. Neurology 1984;34:170-174.

-4 Kawaguchi N, Kuwabara S, Nemoto Y, Fukutake T, Satomura Y, Arimura K, Osame M, Hattori T; Study Group for Myasthenia Gravis in Japan: Treatment and outcome of myasthenia gravis: retrospective multicenter analysis of 470 Japanese patients, 1999-2000. J Neurol Sci 2004;224:43-47.

-5 Pascuzzi RM, Coslett HB, Johns TR: Long-term corticosteroid treatment of myasthenia gravis: report of 116 patients. Ann Neurol 1984;15:291-298.

6 Nagane Y, Suzuki S, Suzuki N, Utsugisawa K: Early aggressive treatment strategy against myasthenia gravis. Eur Neurol 2011;65:16-22.

7 Sathasivam S: Steroids and immunosuppressant drugs in myasthenia gravis. Nat Clin Pract Neurol 2008;4:317327.

-8 Skeie GO, Apostolski S, Evoli A, Gilhus NE, Hart IK, Harms L, Hilton-Jones D, Melms A, Verschuuren J, Horge HW: Guidelines for treatment of autoimmune neuromuscular transmission disorders. Eur J Neurol 2010;17:893-902. 